\title{
Supporting Material: \\ Understanding Nano-Impact Current Spikes: \\ Electrochemical Doping of Impacting Nanoparticles
}

\author{
Enno Kätelhön, Ann Feng, Wei Cheng, Shaltiel Eloul, \\ Christopher Batchelor-McAuley, and Richard G. Compton \\ Department of Chemistry, Physical and Theoretical Chemistry Laboratory, Oxford University, South \\ Parks Road, Oxford, OX1 3QZ, United Kingdom
}

\section{Supporting Material A: Analytical}

\section{Determination of the spatiotemporal concentration profile}

We assume that the concentration $c$ can be expressed as the product of a time- and a spacedependent function:

$$
c(r, t)=v(t) \cdot u(r)
$$

and employ the method of the separation of variables. Setting the free constant to $-\lambda^{2}$ (see reference $^{1}$ ) leads to:

$$
\frac{1}{D} \frac{v^{\prime}}{v}=\frac{1}{u}\left(u^{\prime \prime}+\frac{2}{r} u^{\prime}\right)=:-\lambda^{2} \text { with } \lambda \in \mathbb{R}
$$

where $v^{\prime}, u^{\prime}$, and $u^{\prime \prime}$ represent the temporal and spatial derivatives of $v$ and $u$, respectively. The problem is then solved by the integration of two ordinary differential equation in $r$ and $t$.

\section{Temporal evolution}

In order to determine the time evolution of the concentration, we integrate the left term of Equation (2), which we previously set equal to $-\lambda^{2}$ :

$$
v^{\prime}=-D \lambda^{2} v
$$

This immediately provides the desired time-dependency:

$$
v=k \cdot e^{-D \lambda^{2} t}
$$


where $k$ is an integration constant and $k \in \mathbb{R}$.

\section{Spatial evolution}

For the spatial dependency of $c$, we integrate the space-dependent term in Equation (2):

$$
r^{2} u^{\prime \prime}+2 r u^{\prime}+\lambda^{2} r^{2} u=0
$$

As discussed by Bronstein et al. ${ }^{1}$, the solutions of ordinary differential equations of the form:

$$
r^{2} u^{\prime \prime}+r p(r) u^{\prime}+q(r) u=0
$$

can be developed in convergent series featuring the structure:

$$
u=r^{a} \sum_{i=0}^{\infty} a_{i} r^{i}
$$

where the coefficients $a_{i}$ are real numbers, $a_{i} \in \mathbb{R}$. The parameter $a$ is thereby determined by the defining equation:

$$
a(a-1)+p(0) a+q(0)=0
$$

and the coefficients $a_{i}$ can be evaluated via the method of undetermined coefficients. For the here-investigated problem, this equation is solved by two values, $a=3$ and $a=0$. For $a=3$, the substitution of Equation (7) in Equation (5) manuscript yields:

$$
\sum_{i=0}^{\infty} a_{i}(i+3)(i+2) r^{i+3}+\sum_{i=0}^{\infty} 2 a_{i}(i+3) r^{i+3}+\lambda^{2} \sum_{i=0}^{\infty} a_{i} r^{i+5}=0
$$

The comparison of the terms comprising equal exponents of $r$ provides relations for the individual coefficients:

$$
\lambda^{2} a_{i}+a_{i+2}(i+5)(i+6)=0 \text { with } i \in \mathbb{N}^{0}
$$

and:

$$
a_{i+2}(i+3)(i+4)=0 \text { with } i \in[0,1]
$$

Since according to Equation (11) the coefficients $a_{0}$ and $a_{1}$ hence both must equal zero, Equation (10) solely yields the trivial solution $a_{i}=0$ and therefore $u=0$. For $a=0$, the substitution of Equation (7) in (5) provides:

$$
\sum_{i=0}^{\infty} a_{i} i(i-1) r^{i}+\sum_{i=0}^{\infty} 2 a_{i} i r^{i}+\lambda^{2} \sum_{i=0}^{\infty} a_{i} r^{i+2}=0
$$


The comparison of the different terms reveals that the coefficient $a_{1}$ must equal zero and provides the following relation for the coefficients $a_{i}$ with $i>0$ :

$$
\lambda^{2} a_{i}+a_{i+2}(i+2)(i+3)=0 \text { with } i \in \mathbb{N}^{0}
$$

As $a_{1}$ equals zero, all uneven coefficients must equal zero as well. This equation shows that for a given value of $a_{0}$, all other even coefficients can be calculated via:

$$
a_{i}=(-1)^{\frac{i}{2}} \frac{a_{0} \lambda^{i}}{(i+1) !} \text { with } i \in \mathbb{N}_{\text {even }}^{+}
$$

The solution for $u$ is hence given by:

$$
\begin{aligned}
u & =\sum_{k=0}^{\infty}(-1)^{k} \frac{a_{0}}{(2 k+1) !}(\lambda r)^{2 k} \\
& =\frac{a_{0}}{\lambda r} \sum_{k=0}^{\infty}(-1)^{k} \frac{(\lambda r)^{2 k+1}}{(2 k+1) !}
\end{aligned}
$$

Using the series expansion of the sine function ${ }^{1}$, this expression can be simplified to:

$$
u=a_{0} \frac{\sin (\lambda r)}{\lambda r}
$$

\section{Implementation of boundary conditions}

We calculate the solutions of diffusion equation (4) in the main manuscript by substitution of the spatiotemporal dependencies (4) and (16) into the ansatz function (1) and obtain:

$$
c(r, t)=k_{n} \frac{\sin (\lambda r)}{r} e^{-D \lambda^{2} t}
$$

where we combine $k, a_{0}$, and $\lambda^{-1}$ in the parameter $k_{n} \in \mathbb{R}$. Considering that the sine function approximates a linear function near $r=0$, it is shown that all solutions generally satisfy the boundary condition (3) in the main manuscript, which ensures that the concentration gradient vanishes at $r=0$ :

$$
\left.\left.\frac{\partial}{\partial r} \frac{\sin (\lambda r)}{r}\right|_{r=0} \stackrel{r \rightarrow 0}{\approx} \frac{\partial}{\partial r} \frac{\lambda r}{r}\right|_{r=0}=0
$$

We further identify solutions that fulfil the boundary condition (2) in the main manuscript, which sets the concentration at the particle surface to a constant value. Since the concentration at the interface remains constant at any time, we further analyse Equation (17) and determine all values for $\lambda$ that lead to a time-invariant concentration at $r_{0}$. A closer investigation of the general solution reveals that such functions generally satisfy the following condition: 


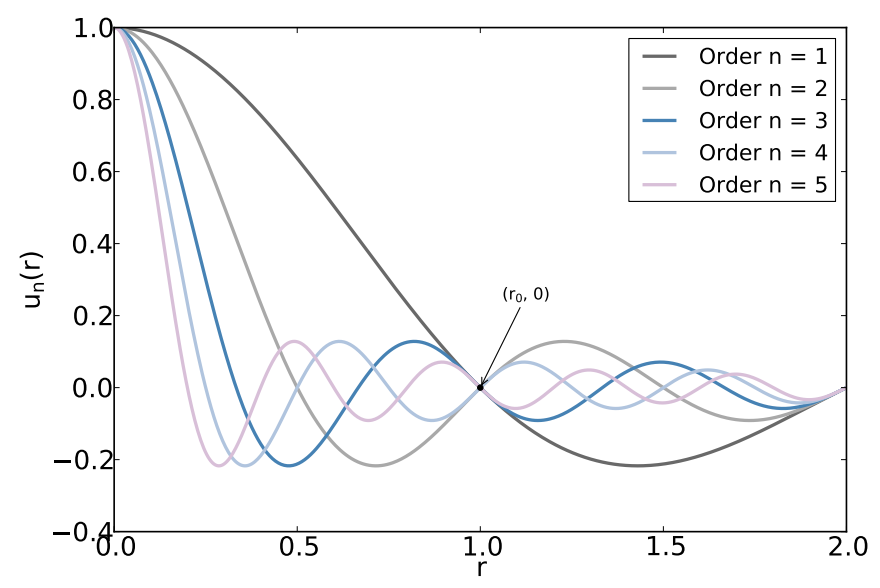

Figure S1: General solutions $u_{n}$ plotted for various orders $n . a_{0}, r_{0}$ and $c^{*}$ are set to unity.

$$
u_{n}\left(r_{0}\right)=0 \Leftrightarrow \lambda_{n}=\frac{n \pi}{r_{0}} \text { with } n \in \mathbb{N}^{+}
$$

where $n$ represents the order of the solution. The so-obtained results for $u_{n}$ are shown in Figure S1. Since the desired solutions must fulfil boundary condition (2) in the main manuscript, we further exploit the linearity and the homogeneity of the diffusion equation and offset the overall solution for $c$, which is a superposition of all individual solutions (17) with (19), by $c^{*}$ :

$$
c(r, t)=c^{*}+\sum_{n=1}^{\infty} k_{n} \frac{\sin \left(\lambda_{n} r\right)}{r} e^{-D \lambda_{n}^{2} t}
$$

This is a representation of a Fourier series, whose coefficients $k_{n}$ are calculated via the following equation $^{2}$ :

$$
\begin{aligned}
k_{n} & =\frac{2}{r_{0}} \int_{0}^{r_{0}} d r r^{2} \frac{\sin \left(\lambda_{n} r\right)}{r} \cdot c(r, t=0) \\
& =\frac{2}{r_{0}} \int_{0}^{r_{0}} d r r^{2} \frac{\sin \left(\lambda_{n} r\right)}{r} \cdot\left(-\Theta\left(r_{0}-r\right) c^{*}\right) \\
& =-\frac{2 c^{*}}{r_{0}} \int_{0}^{r_{0}} d r r \sin \left(\lambda_{n} r\right) \\
& =(-1)^{n} \frac{2 r_{0} c^{*}}{n \pi} \\
& =(-1)^{n} \frac{2 c^{*}}{\lambda_{n}}
\end{aligned}
$$

where $\Theta$ is the Heaviside step function. Equation (20) hence transformes into the following expression, which matches the initial concentration profile at $t=0$ : 


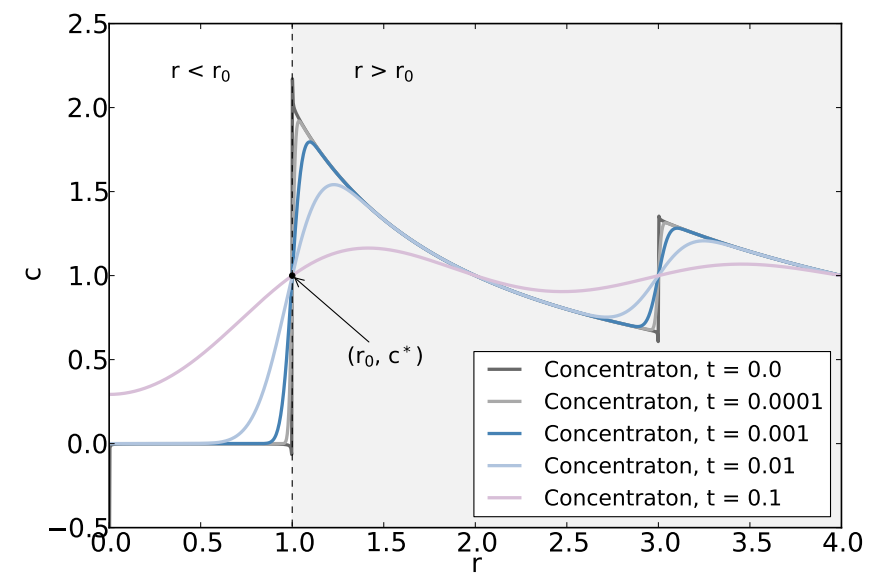

Figure S2: Temporal evolution of the concentration profile. $D, r_{0}$, and $c^{*}$ are set to unity and the first 1000 terms of the sum are evaluated. Please note that the concentration profile is only meaningful in the range $r<r_{0}$, function values for greater $r$ are solely depicted for illustration.

$$
c(r, t)=c^{*}+2 c^{*} \sum_{n=1}^{\infty}(-1)^{n} \frac{\sin \left(\lambda_{n} r\right)}{\lambda_{n} r} e^{-D \lambda_{n}^{2} t}
$$

The resultant temporal evolution of the concentration profile is shown in Figure S2, where the values of the parameters $D, r_{0}$, and $c^{*}$ are all set to unity.

\section{Analytical solution for the current transient}

Substitution of the concentration profile (22) in Equation (5) in the main manuscript yields the desired expression for the current $I_{\text {anal }}$ as a function of time:

$$
\begin{aligned}
I_{\text {anal }}(t) & =-\frac{d}{d t} \int_{r<r_{0}} d V q_{0} c(\vec{r}, t) \\
& =-\frac{d}{d t} \int_{0}^{r_{0}} d r 4 \pi r^{2} q_{0} c(\vec{r}, t) \\
& =-8 \pi c^{*} q_{0} \sum_{n=1}^{\infty}(-1)^{n} \int_{0}^{r_{0}} d r \frac{r \sin \left(\lambda_{n} r\right)}{\lambda_{n}} \frac{d}{d t} e^{-D \lambda_{n}^{2} t} \\
& =8 \pi c^{*} D q_{0} \sum_{n=1}^{\infty}(-1)^{n} \lambda_{n} e^{-D \lambda_{n}^{2} t} \int_{0}^{r_{0}} d r r \sin \left(\lambda_{n} r\right) \\
& =-8 \pi c^{*} D q_{0} r_{0} \sum_{n=1}^{\infty}(-1)^{n} e^{-D \lambda_{n}^{2} t} \cos (n \pi) \\
& =-8 \pi c^{*} D q_{0} r_{0} \sum_{n=1}^{\infty} e^{-D \lambda_{n}^{2} t}
\end{aligned}
$$




\section{Supporting material B: Experimental}

\section{Characterisation of the potentiostat}

In order to quantify the impact of the electronic circuitry and analogue-digital conversion of the employed potentiostat (Metrohm microAutolab Type II) on the measured signal, we determined the impulse response of the potentiostat. To this end, the connectors for the reference- and the working electrode were connected via a $100 \mathrm{M} \Omega$ resistor while the connectors for the counterand the reference electrode were short cut. Rectangular voltage pulses featuring a duration of $100 \mu$ s and various heights were applied at the working electrode via the internal digital-analogue converter of the potentiostat and the current response of the working electrode was measured through the internal current amplifier and analogue-digital converter.

The shape of the recorded spikes may hence be influenced by the transfer functions of the digital-analogue converter and amplifier of the applied pulse, the resistor, and the current amplifier and analogue-digital converter. We however assume that the limiting element will be the current amplifier and consequently use the impulse response recorded via all three elements to approximate the potentiostat transfer function as discussed in the following section.

\section{Supporting material C: Computational}

\section{Computational model of the filter response}

We determine the transfer function $H$ of the potentiostat on the basis of the potentiostat's current response to an applied voltage pulse, see Supporting Material B:

$$
H(s)=\frac{\mathcal{L}(y(t))}{\mathcal{L}(x(t))}
$$

where $s$ is the Laplace parameter, $\mathcal{L}$ the Laplace transformation, and $x(t)$ and $y(t)$ the inputand output-signals of the filter as a function of time, respectively.

Figure S3 presents the measured spike shapes that result form the voltage pulses featuring various pulse heights and a constant pulse length. We find that spike heights scale with the height of the applied pulses though the spike shapes remain unaffected suggesting that the system may be described well by a transfer function of the form of a linear time-invariant filter. This invariance of the spike shape at varied pulse heights can be seen even clearer in an overlay plot of the normalised spikes as it is shown in Figure S4: Aside from the different noise characteristics that arise from the digital nature of the potentiostat's internal analogue digital converter, the shapes are found to be identical.

The unexpected sharp onset feature of the spikes scales with the height of the applied voltage pules though it seems to remain unaffected by the bandwidth limitations of the analogue measurement circuitry. We therefore attribute this effect to unwanted crosstalk between the working- and reference- or counter electrode. The applied voltage pulse appears to partly leak 


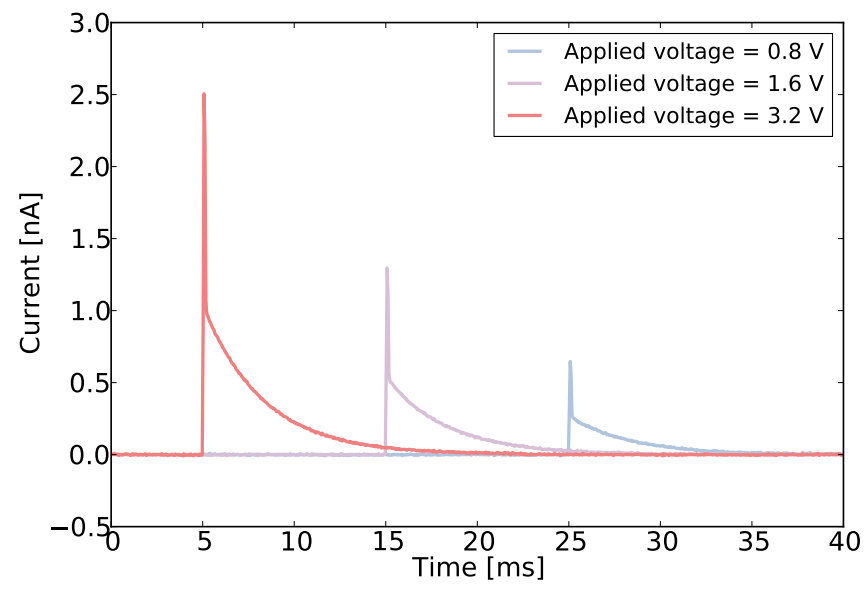

Figure S3: Spike shapes recorded as the potentiostat's response to voltage pulses at varied pulse heights. During the experiment, counter- and reference electrode were short cut and connected via a $100 \mathrm{M} \Omega$ resistor. Pulses were applied via the internal digital-analogue converter of the potentiostat and featured a pulse length of $100 \mu \mathrm{s}$.

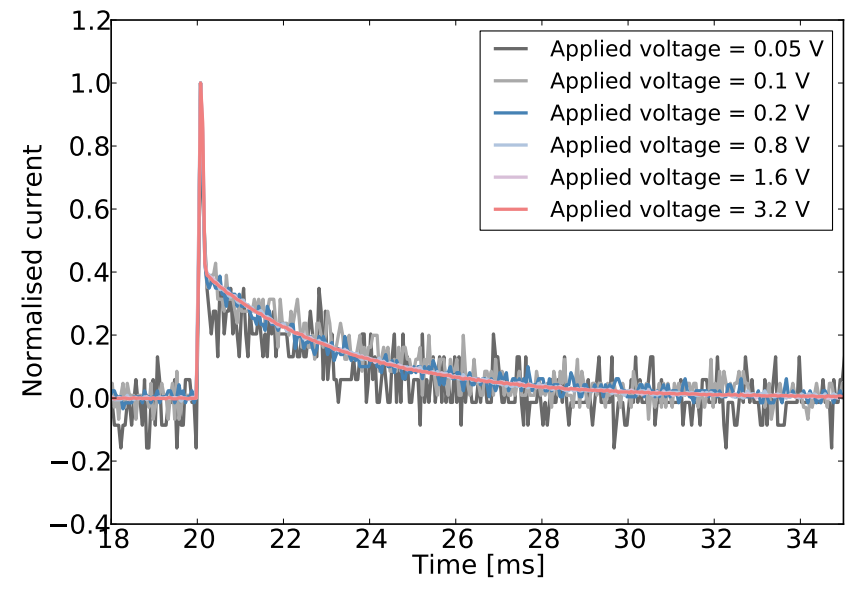

Figure S4: Comparison of the spikes recorded at varied pulse heights. The presented spikes are offset corrected and the peak heights are normalised to unity.

or capacitively couple into the recording channel.

The resistance of the resistor that we use to connect reference- and working electrode is measured via the application of a longer pulse (see Figure S5), which allows the measurement of steady-state currents that are not affected by any bandwidth limitations. The ohmic resistance $R$ is found to be $88 \mathrm{M} \Omega$, which is within the specification provided by the manufacturer.

On the basis of this measurement we can compare the expected and the actually transferred charge during a pulse. To this end, we calculate the charge that is expected pass the resistor within the duration of the applied pulse: 


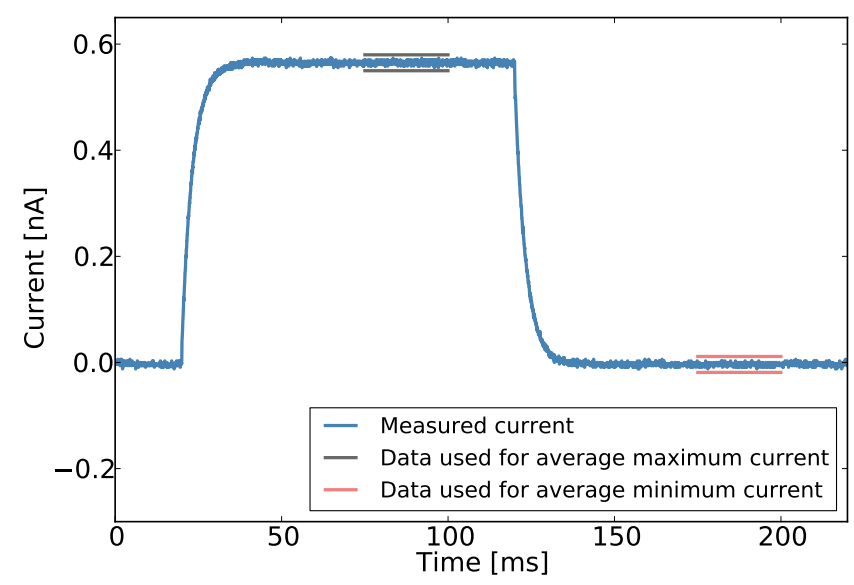

Figure S5: Measurement of the resistance between the working- and the reference electrode. The two highlighted regions highlight the samples that were averaged to determine the ohmic resistance.

$$
Q=\Delta t \frac{U}{R}
$$

where $\Delta t$ is the duration of the pulse, $U$ the applied voltage and $R$ the ohmic resistance of employed resistor, and plot these values with the integrated current of the measured spikes, see Figure S6. Both values are in good agreement suggesting that the overall charge transferred is conserved by the circuitry, though deviations can be found for small transferred charges, where the measurement is limited by the resolution of the potentiostat's analogue-digital converter, see Figure S4. The value of the lowest charge measurement is not depicted in the logarithmic plot, since due to the poor bit resolution in this current regime we here measured a negative transferred charge.

In order to find the transfer function of the analogue circuitry, we compare the measured spikes to impulse responses of various filter models. Herein, we find that a first-order low-pass filter operated at a cutoff frequency $f_{c}$ of $48 \mathrm{~Hz}$,

$$
H(s)=\frac{1}{\frac{s}{2 \pi f_{c}}+1}
$$

shows excellent agreement with all experimental data, though the gain had to be adjusted in the range of about 0.92 to 0.98 to compensate for variations in the overall transferred charge. We however assume that these small deviations are due to the crosstalk between the referenceand the working electrode, which may cause the initial sharp spike at the onset and only occurs at fast changes in the potential. During the here-discussed electrochemical measurements, the potential is however kept constant at all time and such deviations are not expected to occur. In the following, we therefore model the filter using a gain of 1.0. Figure S7 shows excellent 


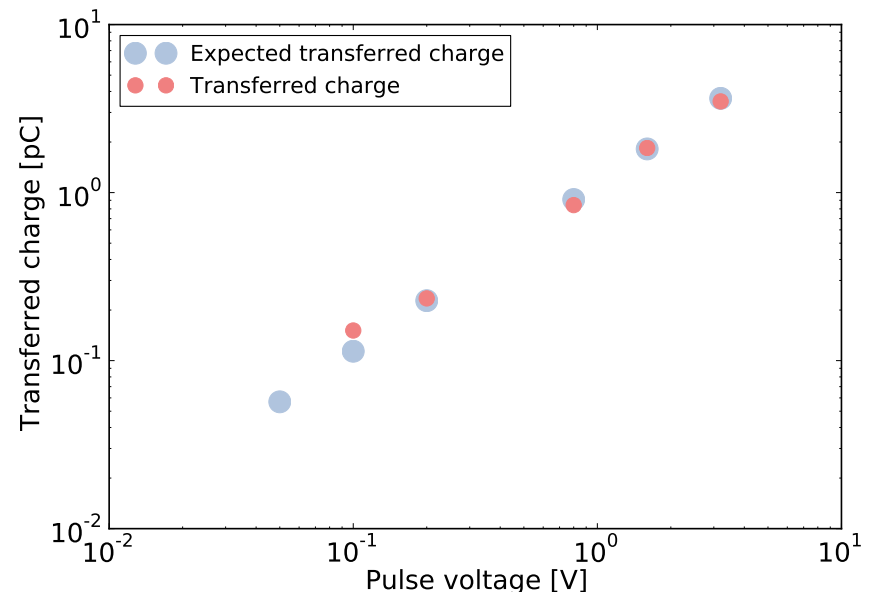

Figure S6: Comparison of the transferred charge that we expected on the basis of the characterisation of the resistor and the applied pulse, and the actually transferred charge found via the numerical integration of the spikes.

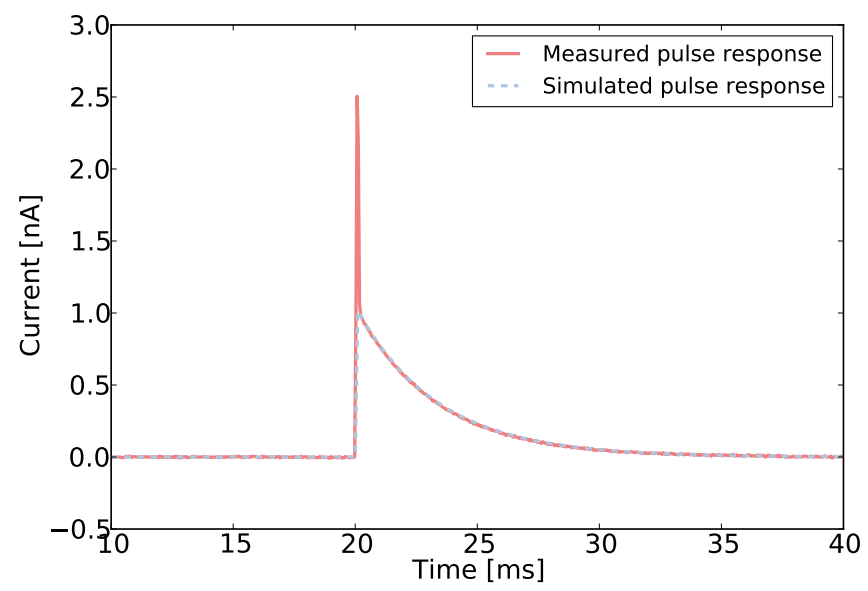

Figure S7: Comparison of the measured potentiostat response to a $3.2 \mathrm{~V}$ pulse and the impulse response of a first-order low-pass filter operated at $48 \mathrm{~Hz}$ and a gain of 0.94 . The filter response is calculated at a sampling frequency of $1 \mathrm{MHz}$ and no oversampling was used. The impulse response is calculated as the response to a $100 \mu$ s pulse and

agreement between the filter model and the experimentally-found spike.

For the later application of the filter model to impulses or analytical solutions for the spike shape, we develop two software algorithms in Python and $C++$, respectively, that transform lists of time-discrete current measurements according to the potentiostats transfer function. To this end, we determine the time-discrete transfer function as discussed previously ${ }^{3}$. In short, Equation (26) is transformed into z-space by conformal mapping via the bilinear transformation and the corresponding linear constant-coefficient difference equation (LCCD) is evaluated. The faster implementation in $C++$, which we exclusively employ in the spike fitting procedure, 
additionally includes an oversampling function: In order to increase the difference between the filter cutoff frequency and the Nyquist frequency of the time-discrete input signal, the signal is up-sampled via linear interpolation before the filter is applied and subsequently down-sampled.

It is noted that the influence of the capacitance of the electrode-electrolyte interface on the eventually measured signal is not considered and assumed to be negligible, which is well supported by a previous study ${ }^{3}$. Therein we demonstrate that in a similar experimental set-up fast reactions in nanoimpact experiments lead to almost ideal filter impulse responses.

\section{Finite-differences simulations}

Using the implicit finite-differences method ${ }^{4 ; 5}$, we numerically simulate the model of ionic diffusion into a sphere, which was introduced above in Section 2.1. To this end, the diffusion equation (4) in the main manuscript is discretised over a one-dimensional space in spherical coordinates $\left(0<r_{i}<r_{0}\right)$ in $n+1$ grid points that are labelled through the indices $i \in[0, n]$. At the particle centre $r_{i=0}=0$ and at the surface of the particle $r_{i=n}=r_{0}$, we apply the following boundary conditions:

$$
c(\vec{r}, t)=\left\{\begin{array}{l}
\frac{d c}{d r}=0 \text { for } r_{i=0} \\
c^{*} \text { for } r_{i=n}
\end{array}\right.
$$

and the initial concentration is set to zero at every grid point, $r_{i}(t=0)=0$.

We use a homogeneous grid featuring a sufficiently dense spatial grid of $\Delta r=r_{0} \cdot 10^{-5}$. An expending time grid is employed starting at a minimum step width of $\Delta t=10^{-12} s$ at $t=0$. At each point in time, we use the tridiagonal matrix algorithm ${ }^{6}$ to solve a set of concentration equations simultaneously over the one-dimensional space. The current response is calculated through the charge balance of ions in the sphere, as shown in Equation (5) in the main manuscript and discretised as shown below:

$$
\begin{aligned}
I(t) & =-\frac{d}{d t} \int_{r<t_{0}} d V q_{0} c(\vec{r}, t) \\
& =2 \pi q_{0} \sum_{i=0}^{n} r_{i}^{2}\left(c_{i}\left(t_{k+1}\right)-c_{i}\left(t_{k}\right)\right) \Delta r
\end{aligned}
$$

where $k$ is the index of the $k^{t h}$ time step. For the comparison with the analytical solution, the simulation used to model transients at various diffusion coefficients using the parameters specified below in section 4.4 in the main manuscript. The simulation is written in $C++$ and run on a personal computer featuring a $3.2 \mathrm{GHz}$ processor and random access memory capacity of 16 GB. 


\section{Automated spike finding}

For the purpose of this study, we developed an automated spike finding- and integration software in Python and $\mathrm{C}++$. After an automated initial preparation of the experimental data, this software processes data in three analysis steps: It first identifies all spikes, then removes any overlapping spikes and spikes that feature a negative integral, and last determines the charge that is transferred during each of the remaining spikes.

In order to prepare the data for the spike finding algorithm, all recorded currents are initially baseline subtracted. Since, during the first milliseconds of each current measurement, a current transient is observed due to the capacitive charging of the electrode-electrolyte interface and interferes with the spikes caused by nano-impacts, we exclude the first 3000 samples of each data set from our analysis. The current is baseline-subtracted by fitting a tenth-order polynomial via the SciPy scientific computing library and subtraction of the fitted function from the measured values. Subsequently, all current measurements are combined into a single time-current array, which is processed in all further spike analysis and fitting.

In the spike detection algorithm, the software identifies individual spikes on the basis of two necessary criteria. For a new spike to be detected, $n_{\text {above }}$ subsequent samples of the baselinesubtracted current must exceed a given threshold $I_{\text {thres }}$, while at least $n_{\text {below }}$ samples following the previously detected spike feature values below the same threshold. The algorithm then saves the respective sample index $i_{\text {onset }}$ as a course onset position of a potential peak and determines the sample $i_{\text {peak }}$ featuring the highest current values within a range of $n_{\text {range }}$ samples adjacent the detected peak onset position:

$$
i_{\text {peak }}=\max \left[i_{\text {onset }}-\frac{n_{\text {range }}}{2}, i_{\text {onset }}+\frac{n_{\text {range }}}{2}\right]
$$

The so-obtained indices of the peak samples are stored as potential the positions of spike peaks for the later analysis.

The spike integral is calculated as the sum of all samples in a given interval adjacent to the potential spike peak position $i_{\text {peak }},\left[i_{\text {peak }}-n_{-}, i_{\text {peak }}+n_{+}\right]$. Since the current was previously baseline-subtracted, the measured integrals exhibit only little dependency on the choice of the integration interval as long as the interval is greater than the length of the longest spike.

Before all data is stored in a file, the detected potential spikes are checked for overlapping spikes, i.e. spikes that feature overlapping integration intervals. If such potential spikes are detected, both spikes are removed from the analysis. In addition, all spikes that were found to feature a negative integral are neglected in the further data processing.

In this study, we use the parameters given in Table $\mathrm{S} 1$ to analyse the data, which was sampled at a frequency of $2 \mathrm{kHz}$. The experimental data comprises 39 measurements featuring a duration of five seconds each. Out of these 39 measurements, three measurements are excluded from the analysis as the employed fitting procedure leads to significant deviations between the fitted and baseline perceived in a visual inspection, i.e. the value of the baseline current far exceeded the 


\begin{tabular}{ll} 
Parameter & Value \\
\hline$I_{\text {thres }}$ & $8 \mathrm{pA}$ \\
$n_{\text {above }}$ & 1 \\
$n_{\text {below }}$ & 20 \\
$n_{\text {range }}$ & 30 \\
$n_{-}$ & 100 \\
$n_{+}$ & 250
\end{tabular}

Table S1: Parameters of the spike finder algorithm used in this study. Data was sampled at a frequency of $2 \mathrm{kHz}$.

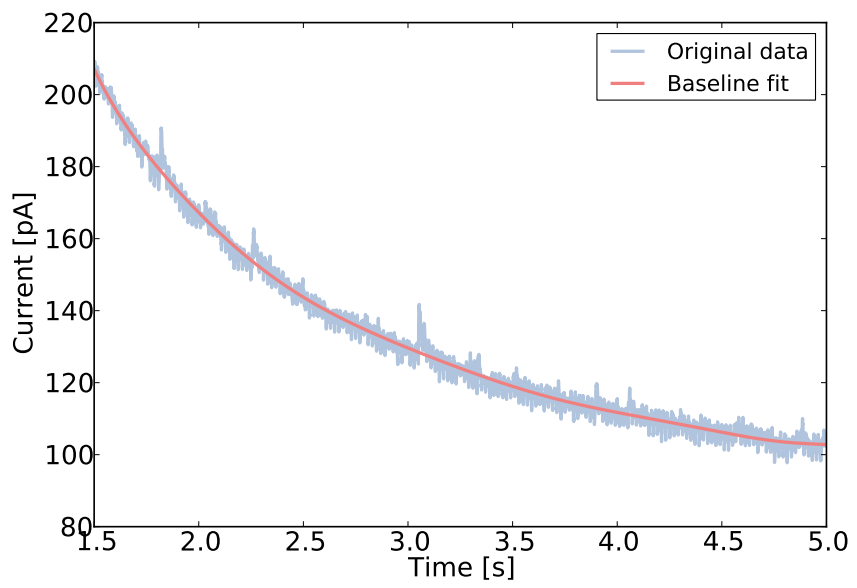

Figure S8: Typical baseline fit to an individual measurement.

value of the experimental data before the onset of a following spike. Figure S8 and S9 present a typical baseline fit and an output generated by the spike finding software, respectively. Using the given set of parameters for the spike detection, a total of 101 spikes are detected, of which 21 are found to overlap. Out of the remaining spikes 80 spikes eight feature a negative spike integral, leaving 72 spikes for the further analysis.

\section{Automated spike fitting}

When fitting experimental data with the above analytical model, three fitting parameters must be considered: The diffusion coefficient $D$ of ions inside the particle, the particle radius $r_{0}$, and the position of the spike onset are all variable, while solely the boundary- and saturation concentration $c^{*}$ of ions and the amount of charge $z e_{0}$ being transferred per ion entering the nanoparticle remain fixed. Since some of these parameters effect the spike shapes in a similar fashion (For example, a greater particle radius and a lower diffusion coefficient both lead to longer decay times) we found that manual fits cannot easily be achieved at an acceptable level of accuracy. We therefore have automated the whole process of comparing experimental and analytical results in order to ensure full reproducibility of the analysis. 


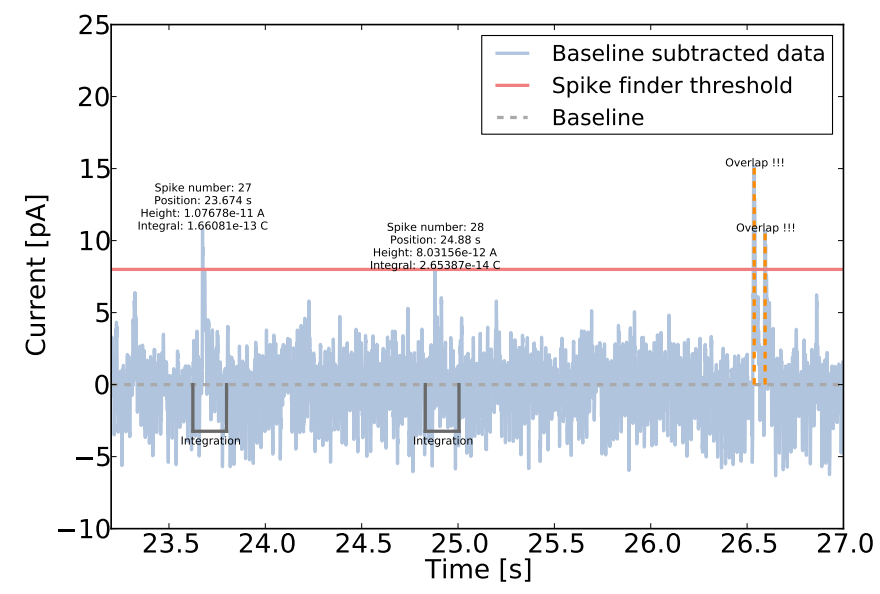

Figure S9: Typical plot generated by the spike finding and integration software. The figure shows a random fragment of the analysed current file after all measurements have been baselinesubtracted and combined into a single current trace.

The employed fitting method is written in $C++$ and based on the least-squares approach. We initially generate parameter maps in $D$ and $r_{0}$ from the input parameters of the fitting algorithm: $n_{D}$ diffusion coefficients are calculated in the range $\left[D_{\min }, D_{\max }\right]$ and $n_{r_{0}}$ particle radii are calculated in the range $\left[r_{0, \min }, r_{0, \max }\right]$, while diffusion coefficients and particle radii feature logarithmic and linear spacings, respectively. In addition to that, $n_{\text {onset }}$ samples preceding each spike peak are considered as possible spike onset positions for the analytical solution. These samples are located at the times $t_{\text {onset }}$ in the experimentally found current, which is sampled at the sampling rate $f_{s, \exp }$.

For every possible combination of the values for $D$ and $r_{0}$, we calculate the analytical solution for the current transient via Equation (23) evaluating the first 30 terms of the infinite sum at a sampling frequency of $f_{s, \text { anal }}=200 \mathrm{kHz}$. We then apply our numerical model of the potentiostat's transfer function at a tenfold over-sampling and subsequently down-sample the result to the sampling frequency $f_{s, \exp }$ of the experimental current $I_{\text {exp }}$ for comparison.

For each spike that is detected by our spike finding algorithm, the function:

$$
F\left(t_{\text {onset }}, D, r_{0}\right)=\sum_{i=0}^{n_{\text {tot }}-1}\left(I_{\text {exp }}\left(\frac{i}{f_{s, \text { exp }}}-t_{\text {onset }}\right)-I_{\text {anal }}\left(\frac{i}{f_{s, \exp }}, D, r_{0}\right)\right)^{2}
$$

is evaluated and the variation problem:

$$
\min \left(F\left(t_{\text {onset }}, D, r_{0}\right),\left\{t_{\text {onset }}, D, r_{0}\right\}\right)
$$

is solved on the basis of the chosen input parameters. If we find that $D=D_{\min }, D=D_{\max }$, $r_{0}=r_{0, \min }$, or $r_{0}=r_{0, \max }$ is the best fit, the respective spike is manually removed from the analysis. Using the parameter values listed in Table $\mathrm{S} 2$ this is the case for one spike, leaving 71 


\begin{tabular}{ll} 
Parameter & Value \\
\hline$n_{\text {onset }}$ & 20 \\
$D_{\text {min }}$ & $10^{-15} \mathrm{~m}^{2} \mathrm{~s}^{-1}$ \\
$D_{\text {max }}$ & $10^{-13} \mathrm{~m}^{2} \mathrm{~s}^{-1}$ \\
$n_{D}$ & 42 \\
$r_{0, \min }$ & $25 \mathrm{~nm}$ \\
$r_{0, \max }$ & $150 \mathrm{~nm}$ \\
$n_{r_{0}}$ & 52 \\
$n_{\text {tot }}$ & 200
\end{tabular}

Table S2: Parameters employed in the spike fitting algorithm.

spikes for the analysis.

\section{Supporting material D: Numerical}

\section{Validation of the analytical solution}

In order to demonstrate the validity of the analytical solution for the above-derived concentration profiles and current transients, we compare our results with finite difference simulations. Five simulations are run using varied diffusion coefficients and obtained data is plotted alongside the corresponding analytical solutions that are calculated for equal parameters. The respective plots can be found in Figure S10, where both approaches exhibit excellent agreement. It is however noted that for better comparability, we here evaluate the analytical solution numerically at a high sampling frequency of $1 \mathrm{MHz}$ and under consideration of the first 5000 terms in the current expression though lower values are used in the rest of the study. The accuracy of the numerical solution is therefore addressed in detail in the following section.

\section{Numerical accuracy of the model}

The calculation of exact numerical values for the analytical solution of the current transient, Equation (23), is vastly complicated by the form and structure of the equation. For a given set of parameters it adopts the form:

$$
I(t) \propto \sum_{n=1}^{\infty} \exp \left(-\frac{t}{n^{2}}\right)
$$

As it can be seen in the equation, the current is expressed in the form of an infinite sum that features a singularity at $t=0$. Since the current is eventually filtered according to the potentiostats transfer function before it is compared with experimental data, the spike shape close to $t=0$ however does not not play a significant role in our analysis. Fast elements such as the singularity will be masked as impulse responses in the measured current, while only the overall charge is conserved and a superposition of impulse responses and lower-bandwidth 


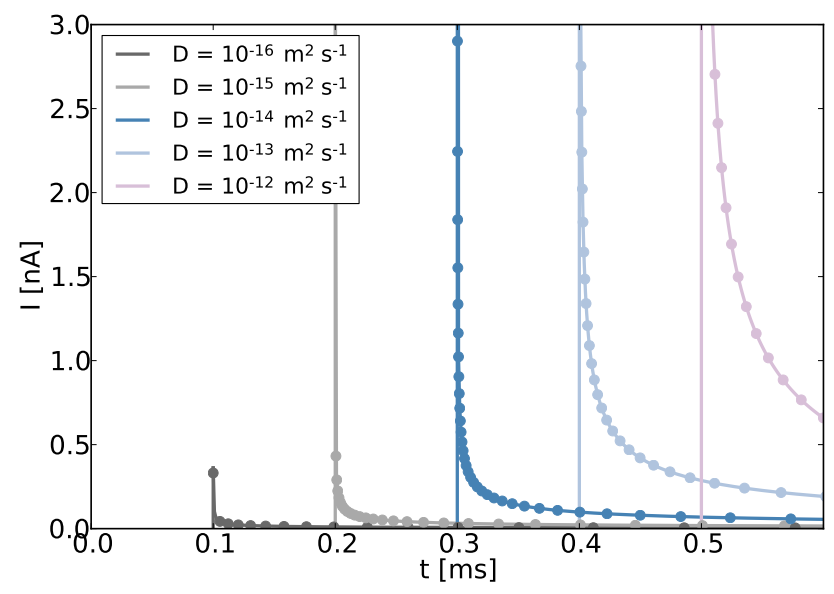

Figure S10: Comparison between the analytical solution and the results obtained from finite difference simulations. Solid lines represent the analytical solution while dots show selected points of the finite difference simulations. The analytical solution uses a sampling rate $f_{s}$ of 1 $\mathrm{MHz}$ and evaluates the first 5000 terms of the infinite sum. All others parameters equal the parameters used in Figure 2 in the main manuscript.

components of the spike remain in the filtered signal. The knowledge of the exact overall charge that is transferred in the numerical solution as well as the corresponding deviations are hence crucial for the later analysis. Herein, two effects that depend on the number of considered terms in the evaluation of the current and the chosen sampling frequency must be considered:

1. The extent, to which the singularity is represented in the numerical calculations, directly depends on the number of terms that are evaluated in the infinite sum. Since all terms equal unity at $t=0$, the evaluation of the sum will lead to exactly the number of evaluated terms. Considering an insufficient number of terms in the numerical solution will hence lead to a reduced overall charge transfer as the contributions of these terms are neglected.

2. In the proximity of $t=0$, we find that the contributions of terms featuring a higher order exhibit significantly faster decays due to the $n^{-2}$ factor in the exponent, while the overall current transient is found to be convex. Due to this convex shape of the transient that occurs adjacent to the singularity, the discretisation of the continuous analytical solution generally leads to an elevated overall charge transfer as compared to the exact value.

We investigate the numerical deviations of spike integrals form the exact values for the above defined parameter ranges of $D \in\left[10^{-15}, 10^{-13}\right] \mathrm{m}^{2} \mathrm{~s}^{-1}$ and $r_{0} \in[25,150] \mathrm{nm}$ with respect to the number of considered terms and the sampling rate. To this end, we first determine the integral for a given number of evaluated terms a high level of numerical accuracy to establish a lower limit for the deviation of the transferred charge. For the numerical integration, we define an expanding grid: 


$$
t_{i}=\sum_{i} \Delta t_{i} \quad \text { with } \quad \Delta t_{i}=\Delta t_{0} \omega^{i}
$$

and calculate the overall transferred charge $Q_{\text {num }}$ via simple rectangular integration:

$$
Q_{\text {num }}=\left|\sum_{i-1} I_{i} \Delta t_{i}\right|
$$

where $I_{i}$ is the $i^{t h}$ sample of the discretised analytical current. The exact value $Q_{\text {theo }}$ that would be obtained for an infinite number of considered terms and an infinite sampling rate can be calculated from the parameters used in the analytical model:

$$
Q_{\text {anal }}=\left|\frac{4}{3} \pi r_{0}^{3} z e_{0} c^{*}\right|
$$

and the percentage deviation of the numerical value from the exact value is determined. We find that for all permutations of the minimal and maximal values of $D$ and $r_{0}$ the deviation is $-2.0 \%$, if we evaluate the first 30 terms using an initial time step of $\Delta t_{0}=10^{-30}$, an expansion factor of $\omega=1.0001$, and an overall integration interval of $10^{2} \mathrm{~s}$.

Next we determine the same values using a fixed sampling rate of $200 \mathrm{kHz}\left(\Delta t_{0}=10^{-5}\right.$ and $\omega=1$ ). Here, we find deviation in the range of $-2.0 \%$ and $7.5 \%$, which can be attributed to the second effect. In the following we use this sampling frequency of $200 \mathrm{kHz}$ and the first 30 terms of the sum for all calculations of Equation (23).

\section{References}

[1] Ilja Nikolajewitsch Bronstein, Konstantin Adolfowitsch Semendjajew, Gerhard Musiol, and Heiner Mühlig. Taschenbuch der Mathematik, 5. Auflage. Verlag Harry Deutsch, 2001.

[2] Rensselaer Polytechnic Institute. Script: Heat conduction in cylindrical and spherical coordinates. http: //www.ewp.rpi.edu /hartford/ ernesto /S2006/CHT /Notes /ch03.pdf, page accessed in June 2016.

[3] Enno Kätelhön, Eden EL Tanner, Christopher Batchelor-McAuley, and Richard G Compton. Destructive nano-impacts: What information can be extracted from spike shapes? Electrochim. Acta, 199:297-304, May 2016.

[4] John Crank. The Mathematics of Diffusion: 2nd Edition. Clarendon Press, 1975.

[5] Richard G Compton, Eduardo Laborda, and Kristopher R Ward. Understanding Voltammetry: Simulation of Electrode Processes. World Scientific, 2013.

[6] Llewellyn Thomas. Elliptic problems in linear differential equations over a network. Watson Scientific Computing Laboratory Report, Columbia University, New York., 1949. 